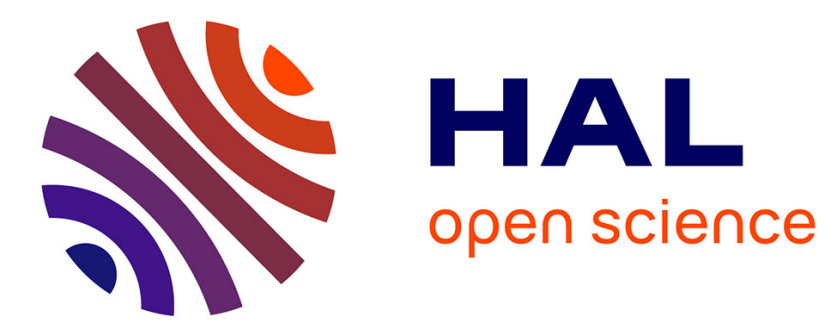

\title{
Therapeutic application of mesenchymal stem cells in osteoarthritis
}

Maxime Ruiz, Stella Cosenza, Marie Maumus, Christian Jorgensen, Danièle Noël

\section{- To cite this version:}

Maxime Ruiz, Stella Cosenza, Marie Maumus, Christian Jorgensen, Danièle Noël. Therapeutic application of mesenchymal stem cells in osteoarthritis. Expert Opinion on Biological Therapy, 2016, 16 (1), pp.33-42. 10.1517/14712598.2016.1093108 . hal-01834251

\section{HAL Id: hal-01834251 \\ https://hal.umontpellier.fr/hal-01834251}

Submitted on 14 Mar 2019

HAL is a multi-disciplinary open access archive for the deposit and dissemination of scientific research documents, whether they are published or not. The documents may come from teaching and research institutions in France or abroad, or from public or private research centers.
L'archive ouverte pluridisciplinaire HAL, est destinée au dépôt et à la diffusion de documents scientifiques de niveau recherche, publiés ou non, émanant des établissements d'enseignement et de recherche français ou étrangers, des laboratoires publics ou privés. 


\title{
Therapeutic application of mesenchymal stem cells in osteoarthritis
}

\author{
Authors \\ Maxime Ruiz ${ }^{\mathrm{a}, \mathrm{b},{ }^{*}, \text { Stella Cosenza }}{ }^{\mathrm{a}, \mathrm{b},{ }^{*}, \text { Marie Maumus }}{ }^{\mathrm{a}, \mathrm{b}}$, \\ Christian Jorgensen ${ }^{\mathrm{a}, \mathrm{b}, \mathrm{c}}$, Danièle Noël $\mathrm{a}^{\mathrm{ab}, \mathrm{c}}$
}

Addresses

anserm, U1183, Hôpital Saint-Eloi, Montpellier, F-34295 France;

bUniversité MONTPELLIER, UFR de Médecine, Montpellier, F-34000 France ;

'Service d'immuno-Rhumatologie, Hôpital Lapeyronie, Montpellier, F-34295 France

*: equally contributing authors

Corresponding author:

Danièle Noël, Inserm U1183, CHRU Saint Eloi,

80 avenue Augustin Fliche, Montpellier, F-34295 France

Tel: +33467330473 - Fax: +33467330113 -E-mail: daniele.noel@inserm.fr 


\section{Therapeutic application of mesenchymal stem cells}

\section{in osteoarthritis}

Introduction: Osteoarthritis is a degenerative disease characterized by cartilage degradation and subchondral bone alterations. This disease represents a global public health problem whose prevalence is rapidly growing with the increasing aging of the population. With the discovery of mesenchymal stem cells (MSC) as possible therapeutic agents, their potential for repairing cartilage damage in osteoarthritis is under investigation.

Areas covered: Characterization of MSCs and their functional properties are mentioned with an insight into their trophic function and secretory profile. We present a special focus on the types of extracellular vesicles that are produced by MSCs and their role in the paracrine activity of MSCs. We then discuss the therapeutic approaches that have been evaluated in preclinical models of osteoarthritis and the results coming out from the clinical trials in patients with osteoarthritis.

Expert Opinion: Mesenchymal stem cell-based therapy seems a promising approach for the treatment of patients with osteoarthritis. Further research is still needed to demonstrate their efficacy in clinical trials using controlled, prospective studies. However, the emergence of mesenchymal stem cell-derived extracellular vesicles as possible therapeutic agents could be an alternative to cell-based therapy.

Keywords: mesenchymal stem cells, trophic factors, extracellular vesicles, regenerative medicine, osteoarthritis 


\section{Introduction}

Diseases affecting the cartilage have an increasing prevalence as people aged or in younger athletes following sport-related injuries. This is related to the poor intrinsic capability of cartilage to regenerate because of the absence of vascularization within the tissue. As the most common form of chronic joint diseases, osteoarthritis (OA) represents a significant public health issue associated with a high economic burden. OA affects more than 20 and 39 million people in United States and Europe respectively, but these numbers are predicted to double until 2020, notably through the increase in average life expectancy and the obesity epidemic [1]. The disease has a huge impact on the patients' quality of life not only related to dysfunction and pain but also to sleep disorder and depression, which further increase the economic burden [2]. Conventional treatments do not cure the disease, at best slightly delay its progression and more generally reduce inflammation and pain. Total joint replacement is the end point surgical option, which is generally successful to alleviate pain and recover motility but represents a substantial risk of infection or thrombosis. Since the early 90's, cellular therapies based on autologous chondrocyte implantation have proved some efficacy to repair lesions following cartilage injuries induced by traumas or early OA [3]. In recent years, the interest of mesenchymal stem/stromal cells (MSC) has been evaluated to circumvent the drawbacks associated with chondrocyte recovery and expansion. Here, we provide an overview of the current knowledge on MSC characteristics and properties as well as their potential therapeutic role in preclinical models of OA and in the clinics.

\section{Physiopathology and current treatments of osteoarthritis}


$\mathrm{OA}$ is a complex disease, which is associated with risk factors such as age, obesity, genetic predisposition, joint instability or trauma. Pain is the predominant symptom with stiffness, and is associated with loss of function of the pathological joint, leading to a drastic reduction in quality of life. Although OA develops over several years, the absence of innervation within the cartilage is associated with delayed symptoms onset, leading to failure in early detection and clinical management of the disease [4]. OA primarily affects knee and hip joints, and less frequently non weight-bearing joints, such as hands or shoulders. The disease is characterized by articular cartilage degradation and osteophyte formation but it also affects other joint tissues, leading to subchondral bone sclerosis and synovial inflammation. Actually while cartilage has long been thought to be responsible for the disease, recent evidence indicates that subchondral bone and synovial tissue are involved in the onset and progression of OA [58].

At the cellular level, pathological changes within the joint affect the chondrocytes, which are the cells responsible for the synthesis and repair of the cartilaginous extracellular matrix (ECM) [9]. These alterations result in a decrease in chondrocyte viability and induce a shift in the balance between anabolic and catabolic activity in favor of the synthesis of matrix metalloproteinases (MMP) and aggrecanases and, cartilage degradation [10]. The altered chondrocytes, which cannot properly respond to mechanical stimulation and synthesize adequate levels of ECM components, enter a vicious cycle in which ECM breakdown dominates synthesis [11].

Current treatments, which are primarily symptomatic, focus on pain relief and inflammatory modulation but do not impact the progressive degeneration of joint tissues [12]. Measures to unload damaged joints by exercise or weight reduction may have a positive effect on pain but non-steroidal anti-inflammatory drugs (NSAIDs) or corticoids are largely used as effective treatments of inflammatory flares of OA. When these strategies fail to alleviate pain, 
surgery is indicated. Osteochondral grafts (mosaicplasty) and microfracture can relieve pain but do not lead to long-term efficacy while joint replacement is effective when pain is associated with disability and radiological deterioration [13]. The efficacy of biotherapies targeting TNF $\alpha$, IL1 $\beta$ or IL6 has also been evaluated, but the results are still disappointing $[14,15]$. Finally, tissue engineering approaches using autologous chondrocyte implantation $(\mathrm{ACI})$ in association or not with matrix $(\mathrm{MACI})$ are routinely applied for the regenerative treatment of injured cartilage and in early OA cartilage lesions [16]. The main effect is however to delay OA but long-term studies in patients with advanced OA are missing. The development of new therapeutic strategies able to prevent the disease progression and regenerate large cartilage lesions are therefore of paramount importance and mesenchymal stem cell-based therapies may be of high interest.

\section{Characteristics and properties of mesenchymal stem cells}

MSCs are adult stem cells that can be isolated from bone marrow (BM-MSCs), adipose tissue (ASCs), umbilical cord, Wharton's jelly, synovium and others [17]. The claim that all MSCs from all tissues are pericytes residing in perivascular location in post-natal organs has been recently discussed [18]. Nevertheless, BM-MSC and ASCs are the two main sources for therapeutic use, with a growing interest for umbilical cord MSCs, which are easy to isolate [17]. The definition for MSCs as proposed by the International Society for Cellular Therapy

(ISCT) relies on three criteria: 1) their adherence to plastic, 2) their phenotype CD105+,

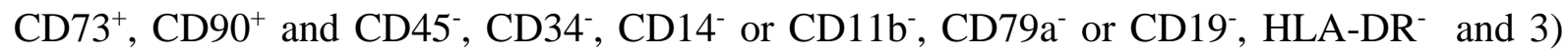
their capacity to differentiate into osteoblasts, adipocytes and chondrocytes [19]. Of interest, the concept and definition of a MSC have been recently reviewed [20]. 
Besides their differentiation potential, MSCs express enzymes and secrete a large number of trophic factors, including growth factors, cytokines, chemokines, which participate to the paracrine activity of these cells [21]. MSCs exert pro-angiogenic activity by acting as pericyte-like cells to support the new vasculature and by secreting factors, such as vascular endothelial growth factor (VEGF), platelet-derived growth factor (PDGF), fibroblast growth factor 2 (bFGF), insulin-like growth factor 1 (IGF-1), hepatocyte growth factor (HGF) or placental growth factor (PIGF). These cells display anti-apoptotic function. Together with VEGF, bFGF, HGF and IGF-1, the secretion of stanniocalcin-1 or transforming growth factor (TGF)- $\beta$ contributes to prevent cells from apoptosis [22]. The production of HGF, bFGF and adrenomedullin by MSCs is also involved in the modulation of fibrosis. HGF might be an important mediator in the anti-fibrotic process by acting on the balance between anti-fibrotic MMPs and tissue inhibitor of MMPs (TIMPs), which play an important role in fibrosis resolution. MSCs are partly resistant to oxidative stress and secrete potent anti-oxidant molecules, namely heme oxygenase (HO)-1 and erythropoietin (EPO). These factors can modulate the production of anti-oxidant molecules in injured tissues, such as superoxide dismutase (SOD) or glutathione peroxidase (GSH-Px) [23].

Finally, MSCs exert anti-inflammatory activity, through the expression of indoleamine 2,3dioxygenase (IDO), and the secretion of several molecules among which, prostaglandin (PG)E2, tumor necrosis factor-inducible gene (TSG)-6, interleukin (IL)-6, HLA-G5 are the main mediators (for review, see [24]. MSCs can impact on the proliferation, differentiation and function of most effector cells of both innate and adaptive immunity. The extent of the immunomodulatory function of MSCs can however differ between samples according to the individual, the species, the tissue source, the culture conditions or the activation status and requires restimulation in culture after freezing preservation procedures. Recommendations for standardization of the assays used to assess the immunoregulatory properties of MSCs have 
been proposed by the ISCT [25]. Consensus on guidelines was obtained for evaluating the immunosuppressive function of MSCs on purified responder cells instead of immune cell populations and interrogating the IDO response as part of an in vitro licensing assay. These recommendations applied to human MSCs. In summary, many of the paracrine functions of MSCs may be of therapeutic interest for reducing cartilage degradation in patients with OA.

\section{Role of extracellular vesicles released by MSCs}

In recent years, the emergence of the role of extracellular vesicles (EVs) as a new way for cell-to-cell communication has rapidly gained much attention. All types of cells release EVs that can interact with other cells in the close environment and transfer functional biomolecules on long distance. EVs are secreted structures surrounded by a phospholipid bilayer and are present in body fluids. There exist different types which can be classified according to their size, composition and biogenesis [26]. The three major types described are exosomes, microparticles and apoptotic bodies. Exosomes are EVs with a size of approximately 80$150 \mathrm{~nm}$ that derive from the endosomal compartment, where membrane invaginates and forms intraluminal vesicles in multivesicular bodies (MVB). Exosomes are then constitutively secreted after fusion of MVBs with the plasma membrane and released in the extracellular space [27]. Exosomes are characterized by tetraspanin proteins (CD9, CD61, CD83) and proteins of the endosomal sorting complex required for transport (ESCRT) (Alix, Tsg101). EVs that are approximately $300-600 \mathrm{~nm}$ in diameter are called microparticles (MP) or microvesicles. They are secreted by budding of the cell membrane after cell stimulation by a stress signal, such as apoptosis, hypoxia, increase of calcium. MPs are characterized by the expression of the membrane markers specific for the cell from which they derive. Apoptotic bodies have a size superior to $1000 \mathrm{~nm}$ and are induced during the late stage of apoptosis as 
blebs of died cells. All EVs are enriched in proteins, lipids and nucleic acids (DNA, mRNA, miRNA, tRNA) that can be delivered to recipient cells, thus contributing to intercellular communication [28]. Even though a selective enrichment of specific molecules into EVs has been described, they display functions that mirror those of their parental cell.

MSCs release EVs that are proposed to be important mediators of the paracrine action in regenerative medicine. The role of MP or exosomes secreted by MSCs is intensively investigated in various animal models and encouraging therapeutic effects have been reported, positioning EVs as a potentially novel alternative to cell-based therapies [29]. However to our knowledge, no literature exists on the possible role of MSC-derived EVs (MSC-EV) in OA. Only one study reports that exosomes from IL1 $\beta$-stimulated synovial fibroblasts could induce OA changes in vitro and in ex vivo models [30]. Since MSCs represent a sub-population of synovial fibroblasts, it can be hypothesized that depending on the environmental signals, the production of exosomes or more generally EVs may be modulated and play a role in the physiopathology of the disease. Nevertheless, MSC-EVs possess functional characteristics that may be of high interest in the treatment of OA.

MSC-EVs have been shown to exert an immunomodulatory effect [31]. MSC-EVs inhibit auto-reactive lymphocyte proliferation, and serve as vehicles for tolerogenic components by induction of regulatory $\mathrm{T}$ cells $[32,33]$. Another study reports that the immunosuppressive effect of MSC-EVs is not directly exerted on T lymphocytes but through the interaction of MSC-EVs with macrophages. Upon interaction, macrophages are induced towards a M2-like phenotype secreting anti-inflammatory cytokines that provide the environment for the generation of a regulatory $\mathrm{T}$ cell population [34]. The effect of MSC-EVs has also been investigated on B cells. An inhibitory effect both on B cell proliferation and differentiation with a reduced secretion of immunoglobulins has been described [35]. Although the reports on the immunoregulatory function of MSC-EVs are still scarce and poorly documented, they 
argue for MSC-EVs as potent modulators of the immune responses and inducers of peripheral tolerance.

The role of MSC-EVs has been largely investigated in many in vivo models including myocardial infarction, brain, lung, liver and acute kidney injuries [29]. In a myocardial infarction model, MSC-EVs protect cardiac tissue from ischemic injury by blood vessel formation, resulting in a significant reduction of the infarct size [36]. In kidney injury, MSCEVs reduce apoptosis, oxidative stress and fibrosis and induce the recovery of renal function [37, 38]. The anti-fibrotic effect of MSC-EVs was also shown on liver by the reduction of collagens I, III and TGF- $\beta 1$ expression and Smad2 phosphorylation [39]. In the hypoxiainduced pulmonary hypertension model, MSC-EVs suppress the hypoxic pulmonary influx of macrophages and the induction of pro-inflammatory mediators. They also suppress hyperproliferative pathways including STAT-3 signaling induced by hypoxia [40]. Finally, in a model of stroke in rats, MSC-EVs contribute to tissue repair by the delivery of miR-133b that enhances neurite outgrowth and functional recovery [41]. Indeed, even though the role of MSC-EVs has not been addressed in osteoarticular diseases, the pro-regenerative, antiapoptotic, anti-fibrotic and anti-inflammatory effects of MSC-EVs as exemplified in the preclinical models described above could justify the interest of using MSC-EVs in OA. The large body of evidence that MSC-EVs exert similar functions as the parental cells provides new perspectives for their use in the treatment of OA.

\section{MSCs-based tissue engineering for OA treatment}

MSCs have been largely used to develop innovative treatments of bone and cartilage disorders including OA. MSCs can either be used as chondroprogenitors to replace injured cartilage in 
tissue engineering approaches or as regenerative cells to stimulate cartilage repair by endogenous cells.

The ability of MSCs to differentiate into chondrocytes in response to several chondrogenic signals such as TGF- $\beta$ superfamily activators and in combination with scaffolds has been reviewed elsewhere $[42,43]$. MSCs can differentiate in vitro into chondrocytes able to secrete the cartilage ECM with properties close to native hyaline articular cartilage. However, stability of the mature chondrocyte phenotype is difficult to achieve while cells tend to undergo hypertrophic differentiation. The source of MSCs is an important issue, as several studies indicate that synovium-derived MSCs and BM-MSCs have higher chondrogenic differentiation potential than other MSC sources [44]. Synovium-derived MSCs differentiate into chondrocytes, which exhibit enhanced expression of specific markers (SOX9, Aggrecan and Collagen 11A1) and higher capacity of proteoglycan synthesis [45]. Even among MSC samples isolated from the same tissue source, there is a vast heterogeneity in the capacity of the cell populations in their trilineage potential [46]. This heterogeneity likely reflects a decrease in the number of true stem cells in the sample. However in term of functionality, no change in chondrogenic potential can be related to age, environmental stresses or disease status [47-49]. A better characterization of homogenous chondroprogenitor populations capable of efficient chondrogenic differentiation is still lacking [50]. Much of the tissue engineering approaches rely on the use of BM-MSCs and develop combinations of cells with scaffolds and growth factors able to support chondrogenic differentiation and form fully functional hyaline articular cartilage. Such strategies are frequently tested in small animal models of surgically induced chondral or osteochondral defects and do not address large defects associated with OA. However, large animal models including sheeps and horses are required for modelling the defects occurring in humans and evaluating the regenerative capacity of MSC-based therapies. The average cartilage thickness in humans is approximately 
2.2-2.5 mm while it is $0.4-1.7 \mathrm{~mm}$ in sheeps and $1.75-2 \mathrm{~mm}$ in horses [51]. In the ovine model, which is anatomically similar to humans, one study reports that implantation of autologous BM-MSCs mixed with chitosan scaffold and TGF- $\beta 3$ resulted in hyaline-like cartilage filling the defects created in the internal groove of the patella [52]. Predifferentiation of BM-MSCs in a collagen gel before implantation allowed better repair than undifferentiated BM-MSC or untreated controls and the efficacy was even enhanced when using triphasic constructs $[53,54]$. The horse is the large animal model with the highest similarity to humans. BM-MSCs loaded on a biphasic sponge scaffold, made of a chondroinductive acid gelatin- $\beta$-tricalcium phosphate (GT) layer and an osteoinductive basic GT underlying layer containing BMP-2, were implanted in osteochondral defects. Higher radiographic, macroscopic and histological scores were recorded with the BM-MSCs loaded on the biphasic sponges [55]. These pre-clinical studies in large animal models do not fulfill all the requirements for evaluating MSC-based therapy for large OA lesions but still indicate improvement of both clinical and functional scores with defects filled with newly hyaline/fibrocartilage on the short or middle term [51].

In the clinics, the proof-of-concept that cell-based therapy could be efficient to restore cartilage function was given in the 90's when Brittberg and collaborators used autologous chondrocytes and demonstrated the efficacy of ACI [56]. Since then, matrix-assisted ACI (MACI) based on different types of scaffolds is in routine use and thousands of patients with osteochondral lesions have been treated [16]. Long term efficacy of the technique is reported primarily on pain relief and fibro/articular cartilage formation, which delays OA. Importantly, some investigations using chondrocytes from end-stage OA patients disclosed similar outcomes as chondrocytes from healthy subjects $[57,58]$. However, the issue of chondrocyte dedifferentiation during culture as well as the need for a more accessible source of cells, with higher expansion potential has prompted the studies on MSCs. The first clinical trial on OA 
patients was performed in 2002 where autologous BM-MSCs within a collagen gel were implanted inside the cartilage lesions under a periosteal flap [59]. Both control and BM-MSCs implanted groups improve functionally but hyaline cartilage was observed only after addition of BM-MSCs. The same group further reported safety and effectiveness of MSC transplantation on the long-term for cartilage repair [60]. In addition when compared to ACI, MSC transplantation was equally effective to relief pain and to improve the patient's quality of life, independently of patient's age. Nevertheless, BM-MSC-based treatment appeared less invasive and reduced both morbidity and operative costs [61]. Similar outcomes with improvement of OA clinical scores were published in additional case reports using BM-MSCs in platelet-rich fibrin glue or collagen $[62,63]$. Finally, a recent investigation comparing the implantation of matrix-induced autologous BM-MSCs versus chondrocytes in 14 patients described significantly better functional outcomes, better knee injury and OA outcome score (KOOS) and visual analog scale score (VAS) with BM-MSCs than chondrocytes [64]. Indeed, although several studies indicate safety and efficacy of MSC-based tissue engineering approaches, no product is available for routine use. A clear demonstration of their interest in larger cohorts of OA patients would be required before they can be used in large scale applications.

\section{Scaffold-free MSC-based therapy in OA patients}

Beyond the capacity of MSCs to repair cartilage after chondrogenic differentiation and implantation in chondral lesions, a growing body of evidence indicates that MSCs can stimulate endogenous cartilage repair through their secretory function, which can modulate local articular environment. Using in vitro chondrocyte coculture models, a number of studies reported that MSCs promote chondrocyte proliferation and stimulate ECM synthesis [65-67]. 
Other reports described the inhibitory effect of MSCs on chondrocyte differentiation $[68,69]$. In our group, we showed that coculture of both BM-MSCs or ASCs with primary chondrocytes isolated from OA patients did not influence the expression of cartilage markers, such as Sox9 or Aggrecan but significantly reduced the expression of fibrotic and hypertrophic markers, which are expressed by OA cartilage [70]. The anti-fibrotic effect of ASCs was mainly associated with the secretion of HGF as demonstrated using neutralizing antibodies that reverted the therapeutic effect of ASCs. We also showed in this coculture system, that ASCs can decrease the camptothecin-induced apoptotic death of chondrocytes. Apart from these properties, MSCs can affect the secretion of inflammatory mediators by chondrocytes and synovial cells that have been isolated from the joint of OA patients. Downregulation of IL-1 $\beta$, IL-6, IL-8 was reported in both cell types and this effect was related to the secretion of PGE2 by ASCs [71]. Similar effect was observed in another study where the expression of IL-1 $\beta$, MMP-1, MMP-13 was decreased in OA synoviocytes while in cartilage, IL1-RA was enhanced upon MSC addition [72]. In addition, a number of factors identified in the secretome of MSCs, namely TGF- $\beta 1$, insulin growth factor (IGF)1, thrombospondin (TSP)-2, stromal-derived factor (SDF)-1, have been shown to favor chondrogenesis in vivo and may be of therapeutic interest for cartilage regeneration [73].

The therapeutic effectiveness of a scaffold-free injection of MSCs has now been validated in numerous pre-clinical models. The first demonstration was published in 2003 by Murphy and collaborators in a caprine model of OA induced by resection of the anterior cruciate ligament and median meniscectomy [74]. Injection of autologous BM-MSCs resulted in regeneration of the medial meniscus, and reduction in osteophyte remodeling, subchondral sclerosis and articular cartilage degradation. Interest of MSC transplantation has been validated in other pre-clinical models of OA [75]. Notably in the murine model of collagenase-induced OA, a dramatic decrease in cartilage degradation, synovial inflammation 
and osteophyte formation was noticed [76]. These impressive results were not observed in the traumatic model of destabilization of the median meniscus (DMM) or in CIOA-induced mice with low synovial inflammation [77]. These findings strongly suggest that inflammationdriven activation of ASCs is required for their protective and immunosuppressive effect in experimental OA. Of note, a beneficial effect of MSC injection was disclosed in horses with OA induced arthroscopically in the middle carpal joint. A significant improvement in PGE2 effusion in synovial fluid was recorded while other parameters did not significantly changed [78]. When evaluated in these models, the survival of exogenously injected MSCs was generally short. We previously reported that intra-articular injection of human ASCs in immunocompromised mice resulted in maintenance of a small percentage of the cells for at least six months [79]. This survival was attributed to the fact that mice could not elicit an adaptive immune response and to the quantity of implanted ASCs, which was very high as compared to similar studies. Nevertheless, when a lower number of human ASCs was infused in immunocompetent naïve mice or mice with CIOA or autoimmune collagen-induced arthritis (CIA), they survived few days. Survival of cells was independent of the healthy or inflammatory environment induced by the disease status but dependent on the route of administration [80].

A number of clinical trials based on the injection of MSCs or ASCs for OA treatment have been initiated or are under way [75]. An updated list of undergoing clinical trials is available in the recent review by Pers and coauthors (Pers et al, in press). A case report has first described cartilage and meniscus growth by MRI, as well as increased range of motion and decreased VAS score after autologous BM-MSC injection in a patient with knee OA [81]. Preliminary studies on 4 or 6 patients with knee OA reported improvement in pain and function after injection of $8-9 \times 10^{6}$ to $20-24 \times 10^{6}$ autologous BM-MSCs [82, 83]. In the first study, patient follow-up at 5 years indicated degradation of the clinical parameters but they 
were still better than at baseline [84]. Safety of BM-MSC implantation was assessed in 227 patients and absence of tumor formation was reported [85]. In a retrospective study where $2 \times 10^{6}$ infrapatellar fat pad-derived MSCs were injected after combination with platelet-rich plasma (PRP), significant reduction of pain as well as increase in function as compared to baseline were reported in the 25 patients with knee OA enrolled [86]. VAS improvement was significantly better in patients with OA of ICRS grade 3 than grade 4 . The results were however not different from the control group, in which the patients had undergone arthroscopic debridement and PRP injection. At 26 months of follow-up, clinical

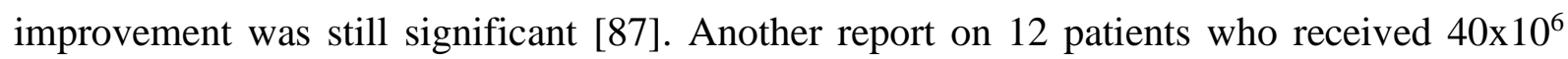
autologous BM-MSCs disclosed improvement of cartilage morphology and quality using MRI T2 mapping suggesting a possible structural benefit of stem cell therapy [88]. In addition in 18 patients with either ankle, hip or knee OA, safety as well as improvement of function and pain were noticed at 12 months post-BM-MSC transplantation, which were no more observed at 30 months follow-up [89]. The effect of intra-articular injection of autologous BM-MSCs three weeks after high tibia osteotomy and microfracture treatment in 28 patients with knee OA was significantly better than in the control group of 28 patients, although both groups improved [90]. A phase I three dose-escalation study on 18 patients and a phase II study with the 9 patients who received the highest dose of $10^{8}$ autologous ASCs demonstrated safety, improvement of the WOMAC score and regeneration of thick hyaline-like cartilage at 6 months [91]. In another phase I dose-escalation study, we also reported improvement in pain and function in 18 patients with knee OA who received autologous ASCs. Interestingly, the best results were obtained with the lowest dose of $2 \times 10^{6}$ ASCs as compared to the doses of 10 $\mathrm{x} 10^{6}$ and $50 \times 10^{6}$ injected cells (Pers et al, submitted). All these studies have demonstrated the safety and tolerability of MSC or ASC injection in patients with knee OA. However, there is an urgent need for randomized, controlled studies. 


\section{Expert opinion}

Continuous growing knowledge on MSC in terms of management of cell isolation and expansion, molecular and functional characterization as well as therapeutic evaluation in preclinical models of many different diseases have pave the way for MSC-based regenerative medicine. MSC-based therapies are relevant for chronic and degenerative disorders in ageing populations, such as OA, where no curative treatments are available. In the present review, we have discussed the two major types of clinical approaches for OA based on either a tissue engineering scaffold-based implantation of MSCs or a scaffold-free direct injection of the stem cells in the injured joint.

The data available from the clinical trials have disclosed the safety of both approaches with no sign of neoplastic proliferation or related side effects. However to date, in contrast to MACI, matrix-induced MSC implantation is not in routine use. One possible reason for that is the inconstant reproducibility of MSC differentiation and uncontrolled in vivo stability of the chondrocyte phenotype. Another reason is the fact that regeneration of large lesions such as those found in patients with high grade OA has not been demonstrated. It is also possible that a better identification of patients who would most benefit for these treatments is required. Profiles of patients have to be drawn based on OA subtypes classified according to the joint involved, the age, the body mass index (BMI), the level of pain, the grade of the disease, the functional limitation, a history of traumas, the genetic susceptibility or the presence of relevant biomarkers. An attempt for such classification is being discussed by the European Society for Clinical and Economic Aspects of Osteoporosis and Osteoarthritis (ESCEO) working group [92]. Such classification of OA subtypes would also benefit for other therapeutic approaches. 
Although preliminary measures of efficacy of the direct injection of MSCs in patients with severe knee OA are encouraging, prospective and placebo-controlled studies are required to determine the effectiveness of this approach. The focus of new clinical trials should address the efficacy of MSC injection in patients with moderate OA and early radiographic stages. In the study by Koh and colleagues, the efficacy of MSC implantation was better in OA patients with grade 3 than with grade 4 [86]. It can be speculated that MSC-based therapy should be more efficient in preventing or limiting the structural progression of the disease at early stages of the disease. Another important issue to be tested is the optimal dose of cells. There are huge differences between cell doses ranging from $2 \times 10^{6}$ to $10^{8}$ cells/joint and discrepancies between clinical trials. We observed the best efficacy on pain and function with $2 \times 10^{6}$ ASCs/knee joint (Pers et al, submitted) whereas Jo and collaborators noticed improvement of pain, function and histology with the highest dose of $10^{8}$ cells/joint [91]. We and others are evaluating this aspect in ongoing clinical trials [93]. Beside the cell dose, the need for repeated injections of MSCs, which should theoretically enhance or prolong the response, has not been investigated. This comes along with the possibility to use autologous MSCs that have been frozen and/or allogeneic MSCs and the requirement to evaluate the host immune response against the injected cells.

The exact mechanism by which MSCs exert their therapeutic efficacy in patients with OA is not known. Indications from the mouse models suggest that the main effector mechanism could be the modulation of inflammation that in turn reduces cartilage degradation. Although differentiation of injected cells into chondrocytes may occur, this is likely not the major mechanism. It is plausible that other mechanisms, such as inhibition of apoptosis or induction of endogenous cell proliferation act in concert with the immunomodulatory effects of MSCs. This points to the notion that MSCs likely act via the secretion of diverse molecules whose overall effect will be difficult to reproduce by a unique molecule but rather, a combination of 
molecules will be needed. This is why the use of EVs isolated from MSC supernatants could be an alternative to cell-based therapies. Since EVs reproduce the functions of the cells from which they originate, they should be tested in cell-free therapeutic approaches in the many applications where MSCs have proven to be effective. Although not yet validated for osteoarticular disease models, our preliminary data suggest that EVs can efficiently reduce histological scores in a murine model of OA (pers. com.). Future experiments are required before MSCs or MSC-derived EVs can be used routinely in the clinic for treating patients with OA.

\section{Declaration of interest}

Work in the laboratory Inserm U1183 was supported by the Inserm Institute and the University of Montpellier. Funding was obtained from the European Community's Horizon 2020 program for the collaborative project: "ADIPOA2: Clinical trial of autologous adipose-derived mesenchymal stromal cells in the treatment of mild to moderate osteoarthritis" (\#: 643809). We are grateful to Arthritis R\&D through the program "ROAD: Research on OsteoArthritis Diseases" and to the Fondation de l'Avenir (grant number AP-RMA-2015-013), Paris-France. We also thank the Agence Nationale pour la Recherche for support of the national infrastructure: "ECELLFRANCE: Development of a national adult mesenchymal stem cell based therapy platform" (ANR-11-INSB-005). The authors have no other relevant affiliations or financial involvement with any organization or entity with a financial interest in or financial conflict with the subject matters or materials discussed in the manuscript. This includes employment, consultancies, honoraria, stock ownership or options, expert testimony, grants or patents received or pending, or royalties. 


\section{Bibliography}

1. Wang Y, Yuan M, Guo QY, Lu SB, Peng J. Mesenchymal stem cells for treating articular cartilage defects and osteoarthritis. Cell Transplant 2014.

2. Gore M, Tai KS, Sadosky A, Leslie D, Stacey BR. Clinical comorbidities, treatment patterns, and direct medical costs of patients with osteoarthritis in usual care: a retrospective claims database analysis. J Med Econ 2011;14:497507.

3. Kristjansson B, Honsawek S. Current perspectives in mesenchymal stem cell therapies for osteoarthritis. Stem Cells Int 2014;2014:194318.

4. Blanco FJ, Ruiz-Romero C. Osteoarthritis: Metabolomic characterization of metabolic phenotypes in OA. Nat Rev Rheumatol 2012;8:130-132.

5. Bondeson J, Wainwright SD, Lauder S, Amos N, Hughes CE. The role of synovial macrophages and macrophage-produced cytokines in driving aggrecanases, matrix metalloproteinases, and other destructive and inflammatory responses in osteoarthritis. Arthritis Res Ther 2006;8:R187.

6. Sellam J, Berenbaum $F$. The role of synovitis in pathophysiology and clinical symptoms of osteoarthritis. Nat Rev Rheumatol 2010;6:625-635.

7. Prasadam I, Crawford R, Xiao Y. Aggravation of ADAMTS and matrix metalloproteinase production and role of ERK1/2 pathway in the interaction of osteoarthritic subchondral bone osteoblasts and articular cartilage chondrocytes -- possible pathogenic role in osteoarthritis. J Rheumatol 2012;39:621-634.

8. Zhen G, Wen C, Jia X, et al. Inhibition of TGF-beta signaling in mesenchymal stem cells of subchondral bone attenuates osteoarthritis. Nat Med 2013;19:704712.

9. Goldring MB. Update on the biology of the chondrocyte and new approaches to treating cartilage diseases. Best Pract Res Clin Rheumatol 2006;20:1003-1025.

10. Burrage PS, Mix KS, Brinckerhoff CE. Matrix metalloproteinases: role in arthritis. Front Biosci 2006;11:529-543.

11. Bijlsma JW, Berenbaum F, Lafeber FP. Osteoarthritis: an update with relevance for clinical practice. Lancet 2011;377:2115-2126.

12. Hochberg MC, Altman RD, April KT, et al. American College of Rheumatology 2012 recommendations for the use of nonpharmacologic and pharmacologic therapies in osteoarthritis of the hand, hip, and knee. Arthritis Care Res (Hoboken) 2012;64:465-474.

13. Mithoefer K, McAdams T, Williams RJ, Kreuz PC, Mandelbaum BR. Clinical efficacy of the microfracture technique for articular cartilage repair in the knee: an evidence-based systematic analysis. Am J Sports Med 2009;37:2053-2063.

14. Chevalier X, Goupille $P$, Beaulieu AD, et al. Intraarticular injection of anakinra in osteoarthritis of the knee: a multicenter, randomized, double-blind, placebocontrolled study. Arthritis Rheum 2009;61:344-352.

15. Chevalier X, Ravaud $P$, Maheu E, et al. Adalimumab in patients with hand osteoarthritis refractory to analgesics and NSAIDs: a randomised, multicentre, double-blind, placebo-controlled trial. Ann Rheum Dis 2014.

16. Ringe J, Burmester GR, Sittinger M. Regenerative medicine in rheumatic disease-progress in tissue engineering. Nat Rev Rheumatol 2012;8:493-498. 
17. Moroni L, Fornasari PM. Human mesenchymal stem cells: a bank perspective on the isolation, characterization and potential of alternative sources for the regeneration of musculoskeletal tissues. J Cell Physiol 2012;228:680-687.

18. Bianco P. "Mesenchymal" stem cells. Annu Rev Cell Dev Biol 2014;30:677-704.

19. Dominici M, Le Blanc K, Mueller I, et al. Minimal criteria for defining multipotent mesenchymal stromal cells. The International Society for Cellular Therapy position statement. Cytotherapy 2006;8:315-317.

20. Bianco P. Stem cells and bone: a historical perspective. Bone 2015;70:2-9.

21. Maumus M, Jorgensen $C$, Noel D. Mesenchymal stem cells in regenerative medicine applied to rheumatic diseases: role of secretome and exosomes. Biochimie 2013;95:2229-2234.

22. Meirelles Lda S, Fontes AM, Covas DT, Caplan AI. Mechanisms involved in the therapeutic properties of mesenchymal stem cells. Cytokine Growth Factor Rev 2009;20:419-427.

23. de Almeida DC, Donizetti-Oliveira C, Barbosa-Costa P, Origassa CS, Camara NO. In search of mechanisms associated with mesenchymal stem cell-based therapies for acute kidney injury. Clin Biochem Rev 2013;34:131-144.

24. Ghannam S, Bouffi C, Djouad F, Jorgensen C, Noel D. Immunosuppression by mesenchymal stem cells: mechanisms and clinical applications. Stem Cell Res Ther 2010;1:2.

25. Krampera M, Galipeau J, Shi Y, Tarte K, Sensebe L. Immunological characterization of multipotent mesenchymal stromal cells--The International Society for Cellular Therapy (ISCT) working proposal. Cytotherapy 2013;15:1054-1061.

26. Gyorgy B, Hung ME, Breakefield XO, Leonard JN. Therapeutic applications of extracellular vesicles: clinical promise and open questions. Annu Rev Pharmacol Toxicol 2015;55:439-464.

27. Lai RC, Yeo RW, Lim SK. Mesenchymal stem cell exosomes. Semin Cell Dev Biol 2015;40:82-88.

28. Penfornis P, Vallabhaneni KC, Whitt J, Pochampally R. Extracellular vesicles as carriers of microRNA, proteins and lipids in tumor microenvironment. Int $\mathbf{J}$ Cancer 2015.

29. Akyurekli C, Le Y, Richardson RB, Fergusson D, Tay J, Allan DS. A systematic review of preclinical studies on the therapeutic potential of mesenchymal stromal cell-derived microvesicles. Stem Cell Rev 2015;11:150-160.

30. Kato T, Miyaki S, Ishitobi H, et al. Exosomes from IL-1beta stimulated synovial fibroblasts induce osteoarthritic changes in articular chondrocytes. Arthritis Res Ther 2014;16:R163.

31. Bruno S, Deregibus MC, Camussi G. The secretome of mesenchymal stromal cells: Role of extracellular vesicles in immunomodulation. Immunol Lett 2015.

32. Mokarizadeh A, Delirezh N, Morshedi A, Mosayebi G, Farshid AA, Mardani K. Microvesicles derived from mesenchymal stem cells: potent organelles for induction of tolerogenic signaling. Immunol Lett 2012;147:47-54.

33. Blazquez R, Sanchez-Margallo FM, de la Rosa O, et al. Immunomodulatory Potential of Human Adipose Mesenchymal Stem Cells Derived Exosomes on in vitro Stimulated T Cells. Front Immunol 2014;5:556.

34. Zhang B, Yin Y, Lai RC, Tan SS, Choo AB, Lim SK. Mesenchymal stem cells secrete immunologically active exosomes. Stem Cells Dev 2014;23:1233-1244. 
35. Budoni M, Fierabracci A, Luciano R, Petrini S, Di Ciommo V, Muraca M. The immunosuppressive effect of mesenchymal stromal cells on B lymphocytes is mediated by membrane vesicles. Cell Transplant 2013;22:369-379.

36. Bian S, Zhang L, Duan L, Wang X, Min Y, Yu H. Extracellular vesicles derived from human bone marrow mesenchymal stem cells promote angiogenesis in a rat myocardial infarction model. J Mol Med (Berl) 2014;92:387-397.

37. Zhou Y, Xu H, Xu W, et al. Exosomes released by human umbilical cord mesenchymal stem cells protect against cisplatin-induced renal oxidative stress and apoptosis in vivo and in vitro. Stem Cell Res Ther 2013;4:34.

38. Bruno S, Grange C, Deregibus MC, et al. Mesenchymal stem cell-derived microvesicles protect against acute tubular injury. J Am Soc Nephrol 2009;20:1053-1067.

39. Li T, Yan Y, Wang B, et al. Exosomes derived from human umbilical cord mesenchymal stem cells alleviate liver fibrosis. Stem Cells Dev 2013;22:845-854.

40. Lee C, Mitsialis SA, Aslam M, et al. Exosomes mediate the cytoprotective action of mesenchymal stromal cells on hypoxia-induced pulmonary hypertension. Circulation 2012;126:2601-2611.

41. Xin H, Li Y, Buller B, et al. Exosome-mediated transfer of miR-133b from multipotent mesenchymal stromal cells to neural cells contributes to neurite outgrowth. Stem Cells 2012;30:1556-1564.

42. Vinatier $\mathbf{C}$, Bouffi $\mathrm{C}$, Merceron $\mathrm{C}$, et al. Cartilage engineering: Towards a biomaterial-assisted mesenchymal stem cell therapy. Curr Stem Cell Res Ther 2009;4:318-329.

43. Demoor M, Ollitrault D, Gomez-Leduc T, et al. Cartilage tissue engineering: molecular control of chondrocyte differentiation for proper cartilage matrix reconstruction. Biochim Biophys Acta 2014;1840:2414-2440.

44. Li Q, Tang J, Wang R, et al. Comparing the chondrogenic potential in vivo of autogeneic mesenchymal stem cells derived from different tissues. Artif Cells Blood Substit Immobil Biotechnol 2010;39:31-38.

45. Ogata Y, Mabuchi Y, Yoshida M, et al. Purified Human Synovium Mesenchymal Stem Cells as a Good Resource for Cartilage Regeneration. PLoS One 2015;10:e0129096.

46. Russell KC, Phinney DG, Lacey MR, Barrilleaux BL, Meyertholen KE, O'Connor KC. In vitro high-capacity assay to quantify the clonal heterogeneity in trilineage potential of mesenchymal stem cells reveals a complex hierarchy of lineage commitment. Stem Cells 2010;28:788-798.

47. Murphy JM, Dixon K, Beck S, Fabian D, Feldman A, Barry F. Reduced chondrogenic and adipogenic activity of mesenchymal stem cells from patients with advanced osteoarthritis. Arthritis Rheum 2002;46:704-713.

48. Scharstuhl A, Schewe B, Benz K, Gaissmaier C, Buhring HJ, Stoop R. Chondrogenic potential of human adult mesenchymal stem cells is independent of age or osteoarthritis etiology. Stem Cells 2007;25:3244-3251.

49. Dudics V, Kunstar A, Kovacs J, et al. Chondrogenic potential of mesenchymal stem cells from patients with rheumatoid arthritis and osteoarthritis: measurements in a microculture system. Cells Tissues Organs 2009;189:307-316.

50. O'Sullivan J, D'Arcy S, Barry FP, Murphy JM, Coleman CM. Mesenchymal chondroprogenitor cell origin and therapeutic potential. Stem Cell Res Ther 2011;2:8. 
51. Grassel S, Lorenz J. Tissue-engineering strategies to repair chondral and osteochondral tissue in osteoarthritis: use of mesenchymal stem cells. Curr Rheumatol Rep 2014;16:452.

52. Mrugala D, Bony $\mathbf{C}$, Neves $\mathbf{N}$, et al. Phenotypic and functional characterisation of ovine mesenchymal stem cells: application to a cartilage defect model. Ann Rheum Dis 2008;67:288-295.

53. Marquass B, Schulz R, Hepp P, et al. Matrix-associated implantation of predifferentiated mesenchymal stem cells versus articular chondrocytes: in vivo results of cartilage repair after 1 year. Am J Sports Med 2011;39:1401-1412.

54. Marquass B, Somerson JS, Hepp P, et al. A novel MSC-seeded triphasic construct for the repair of osteochondral defects. J Orthop Res 2010;28:15861599.

55. Seo JP, Tanabe T, Tsuzuki N, et al. Effects of bilayer gelatin/beta-tricalcium phosphate sponges loaded with mesenchymal stem cells, chondrocytes, bone morphogenetic protein-2, and platelet rich plasma on osteochondral defects of the talus in horses. Res Vet Sci 2013;95:1210-1216.

56. Brittberg M, Lindahl A, Nilsson A, Ohlsson C, Isaksson O, Peterson L. Treatment of deep cartilage defects in the knee with autologous chondrocyte transplantation. N Engl J Med 1994;331:889-895.

57. Hollander AP, Dickinson SC, Sims TJ, et al. Maturation of tissue engineered cartilage implanted in injured and osteoarthritic human knees. Tissue Eng 2006;12:1787-1798.

58. Kreuz PC, Muller S, Ossendorf C, Kaps C, Erggelet C. Treatment of focal degenerative cartilage defects with polymer-based autologous chondrocyte grafts: four-year clinical results. Arthritis Res Ther 2009;11:R33.

59. Wakitani S, Goto T, Pineda SJ, et al. Mesenchymal cell-based repair of large, full-thickness defects of articular cartilage. J Bone Joint Surg Am 1994;76:579592.

60. Wakitani S, Okabe T, Horibe S, et al. Safety of autologous bone marrow-derived mesenchymal stem cell transplantation for cartilage repair in 41 patients with 45 joints followed for up to 11 years and 5 months. J Tissue Eng Regen Med 2011;5:146-150.

61. Nejadnik H, Hui JH, Feng Choong EP, Tai BC, Lee EH. Autologous bone marrow-derived mesenchymal stem cells versus autologous chondrocyte implantation: an observational cohort study. Am J Sports Med 2010;38:11101116.

62. Haleem AM, Singergy AA, Sabry D, et al. The Clinical Use of Human CultureExpanded Autologous Bone Marrow Mesenchymal Stem Cells Transplanted on Platelet-Rich Fibrin Glue in the Treatment of Articular Cartilage Defects: A Pilot Study and Preliminary Results. Cartilage 2010;1:253-261.

63. Kasemkijwattana C, Hongeng S, Kesprayura S, Rungsinaporn V, Chaipinyo K, Chansiri K. Autologous bone marrow mesenchymal stem cells implantation for cartilage defects: two cases report. J Med Assoc Thai 2011;94:395-400.

64. Akgun I, Unlu MC, Erdal OA, et al. Matrix-induced autologous mesenchymal stem cell implantation versus matrix-induced autologous chondrocyte implantation in the treatment of chondral defects of the knee: a 2-year randomized study. Arch Orthop Trauma Surg 2015;135:251-263.

65. Wu L, Leijten JC, Georgi N, Post JN, van Blitterswijk CA, Karperien M. Trophic effects of mesenchymal stem cells increase chondrocyte proliferation and matrix formation. Tissue Eng Part A 2011;17:1425-1436. 
66. Wu L, Prins HJ, Helder MN, van Blitterswijk CA, Karperien M. Trophic Effects of Mesenchymal Stem Cells in Chondrocyte Co-Cultures are Independent of Culture Conditions and Cell Sources. Tissue Eng Part A 2012;18:1542-1551.

67. Wang M, Rahnama R, Cheng $T$, et al. Trophic stimulation of articular chondrocytes by late-passage mesenchymal stem cells in coculture. J Orthop Res 2013;31:1936-1942.

68. Lee CS, Burnsed OA, Raghuram V, Kalisvaart J, Boyan BD, Schwartz Z. Adipose stem cells can secrete angiogenic factors that inhibit hyaline cartilage regeneration. Stem Cell Res Ther 2012;3:35.

69. Xu L, Wang Q, Xu F, Ye Z, Zhou Y, Tan WS. Mesenchymal stem cells downregulate articular chondrocyte differentiation in noncontact coculture systems: implications in cartilage tissue regeneration. Stem Cells Dev 2013;22:1657-1669.

70. Maumus M, Manferdini C, Toupet K, et al. Adipose mesenchymal stem cells protect chondrocytes from degeneration associated with osteoarthritis. Stem Cell Res 2013;11:834-844.

71. Manferdini C, Maumus M, Gabusi E, et al. Adipose-derived mesenchymal stem cells exert antiinflammatory effects on chondrocytes and synoviocytes from osteoarthritis patients through prostaglandin e2. Arthritis Rheum 2013;65:12711281.

72. van Buul GM, Villafuertes E, Bos PK, et al. Mesenchymal stem cells secrete factors that inhibit inflammatory processes in short-term osteoarthritic synovium and cartilage explant culture. Osteoarthritis Cartilage 2012;20:1186-1196.

73. Toh WS, Foldager CB, Pei M, Hui JH. Advances in mesenchymal stem cell-based strategies for cartilage repair and regeneration. Stem Cell Rev 2014;10:686-696.

74. Murphy JM, Fink DJ, Hunziker EB, Barry FP. Stem cell therapy in a caprine model of osteoarthritis. Arthritis Rheum 2003;48:3464-3474.

75. Barry $F$, Murphy M. Mesenchymal stem cells in joint disease and repair. Nat Rev Rheumatol 2013;9:584-594.

76. Ter Huurne M, Schelbergen R, Blattes R, et al. Antiinflammatory and chondroprotective effects of intraarticular injection of adipose-derived stem cells in experimental osteoarthritis. Arthritis Rheum 2012;64:3604-3613.

77. Schelbergen RF, van Dalen S, Ter Huurne M, et al. Treatment efficacy of adipose-derived stem cells in experimental osteoarthritis is driven by high synovial activation and reflected by S100A8/A9 serum levels. Osteoarthritis Cartilage 2014;22:1158-1166.

78. Frisbie DD, Kisiday JD, Kawcak CE, Werpy NM, McIlwraith CW. Evaluation of adipose-derived stromal vascular fraction or bone marrow-derived mesenchymal stem cells for treatment of osteoarthritis. J Orthop Res 2009;27:1675-1680.

79. Toupet K, Maumus M, Peyrafitte JA, et al. Long-term detection of human adipose-derived mesenchymal stem cells after intraarticular injection in SCID mice. Arthritis Rheum 2013;65:1786-1794.

80. Toupet K, Maumus M, Luz-Crawford $P$, et al. Survival and biodistribution of xenogenic adipose mesenchymal stem cells is not affected by the degree of inflammation in arthritis. PLoS One 2015;10:e0114962.

81. Centeno CJ, Busse D, Kisiday J, Keohan C, Freeman M, Karli D. Increased knee cartilage volume in degenerative joint disease using percutaneously implanted, autologous mesenchymal stem cells. Pain Physician 2008;11:343-353. 
82. Davatchi F, Abdollahi BS, Mohyeddin M, Shahram F, Nikbin B. Mesenchymal stem cell therapy for knee osteoarthritis. Preliminary report of four patients. Int J Rheum Dis 2011;14:211-215.

83. Emadedin M, Aghdami N, Taghiyar L, et al. Intra-articular injection of autologous mesenchymal stem cells in six patients with knee osteoarthritis. Arch Iran Med 2012;15:422-428.

84. Davatchi F, Sadeghi Abdollahi B, Mohyeddin M, Nikbin B. Mesenchymal stem cell therapy for knee osteoarthritis: 5 years follow-up of three patients. Int J Rheum Dis 2015.

85. Centeno CJ, Schultz JR, Cheever M, Robinson B, Freeman M, Marasco W. Safety and complications reporting on the re-implantation of culture-expanded mesenchymal stem cells using autologous platelet lysate technique. Curr Stem Cell Res Ther 2010;5:81-93.

86. Koh YG, Choi YJ. Infrapatellar fat pad-derived mesenchymal stem cell therapy for knee osteoarthritis. Knee 2012;19:902-907.

87. Koh YG, Choi YJ, Kwon OR, Kim YS. Second-Look Arthroscopic Evaluation of Cartilage Lesions After Mesenchymal Stem Cell Implantation in Osteoarthritic Knees. Am J Sports Med 2014;42:1628-1637.

88. Orozco L, Munar A, Soler R, et al. Treatment of knee osteoarthritis with autologous mesenchymal stem cells: two-year follow-up results. Transplantation 2014;97:e66-68.

89. Emadedin M, Ghorbani Liastani M, Fazeli R, et al. Long-Term Follow-up of Intra-articular Injection of Autologous Mesenchymal Stem Cells in Patients with Knee, Ankle, or Hip Osteoarthritis. Arch Iran Med 2015;18:336-344.

90. Wong KL, Lee KB, Tai BC, Law P, Lee EH, Hui JH. Injectable cultured bone marrow-derived mesenchymal stem cells in varus knees with cartilage defects undergoing high tibial osteotomy: a prospective, randomized controlled clinical trial with 2 years' follow-up. Arthroscopy 2013;29:2020-2028.

91. Jo CH, Lee YG, Shin WH, et al. Intra-articular injection of mesenchymal stem cells for the treatment of osteoarthritis of the knee: a proof-of-concept clinical trial. Stem Cells 2014;32:1254-1266.

92. Arden N, Richette P, Cooper C, et al. Can We Identify Patients with High Risk of Osteoarthritis Progression Who Will Respond to Treatment? A Focus on Biomarkers and Frailty. Drugs Aging 2015.

93. Gupta PK, Das AK, Chullikana A, Majumdar AS. Mesenchymal stem cells for cartilage repair in osteoarthritis. Stem Cell Res Ther 2012;3:25. 